\title{
Analytical evidence of shear band bifurcations for softening materials
}

\author{
D. Caillerie, R. Chambon and F. Collin
}

\section{Introduction}

Strain localization is a phenomenon occuring very often in anelastic media close to rupture and it is very important to take it into account in most of applications, particularly in geotechnics. Strain localization has been widely studied experimentally, see for instance J . Desrues [4] or J. Desrues and G. Viggiani [5]. It has also been put in evidence in finite element simulations even if it presents mesh dependencies, see Chambon et al. [3] for instance. From a theoretical point of view, the Rice condition [6,7], with some assumptions, enables to determine the onset of strain localization but does not necessarily prove the existence of shear bands.

The purpose of this paper is to establish the existence of shear bands by exhibiting analytical solutions. The studied problem is the deformation of a $2 \mathrm{D}$ thick walled cylinder (an annulus) made of an isotropic hardening/softening "elastic" material and submited to internal and external pressures. The considered constitutive equation is a 2D generalization of that one considered in [2] for 1D problems. More precisely, the hardening/softening behaviour considered in [2] holds true for the deviatoric part of the $2 \mathrm{D}$ constitutive relation in any fixed direction of the deviatoric strain (see section 2). Unlike that of [2] which is affine by parts, the strain stress relation considered in this paper is truly non linear. It is said to be "elastic" because the datum of the strain and not that of its history yields the stress. In fact, this "elastic" behaviour fits into the framework of deformation theory of plasticity and can be thought of as the response of some elasto plastic constitutive equation to a

D. Caillerie and R. Chambon

Laboratoire Sols Solides Structures - Risques, Grenoble Université \& CNRS, BP 53, 38041 GRENOBLE Cedex, FRANCE e-mail: Denis.Caillerie@inpg.fr, e-mail: Rene.Chambon@inpg.fr

F. Collin

Département Argenco - Géomécanique et géologie de l'ingénieur, BAT. B52/3, chemin des Chevreuils, 14000 Liège 1, BELGIUM e-mail: F.Collin@ulg.ac.be 
monotonous loading (see [1]). In the present paper elastic unloading can only occur on the inital elastic curve.

\section{Constitutive equation}

Let $\mathbf{u}$ be the displacement, $\nabla \mathbf{u}$ be its gradient and the symmetrical part $\varepsilon=$ $\frac{1}{2}\left(\nabla \mathbf{u}+\nabla \mathbf{u}^{t}\right)$ of it be the strain.

II being the identity second order tensor, the strain $\varepsilon$ is split in its isotropic and deviatoric part:

$$
\varepsilon=\frac{\operatorname{tr} \varepsilon}{2} \mathbb{I}+\varepsilon^{d} \text { with } \operatorname{tr}^{d}=0
$$

and the constitutive equation reads:

$$
\sigma=\left(\lambda+\mu_{d}\right) \operatorname{tr} \varepsilon \mathbb{I}+2\left(\mu_{m}+\left(\mu_{d}-\mu_{m}\right) \frac{M}{\left\|\varepsilon^{d}\right\|}\right) \varepsilon^{d}
$$

where $\|A\|$ is the norm of the second order tensor A (in components $\|A\|^{2}=A_{i j} A_{i j}$ ) and $M=\min \left[\left\|\varepsilon^{d}\right\|, \frac{C}{\sqrt{2}}\right], C$ being a positive constant.

For $\left\|\varepsilon^{d}\right\| \leq \frac{C}{\sqrt{2}}, M=\left\|\varepsilon^{d}\right\|$ and the constitutive equation reads:

$$
\sigma=\left(\lambda+\mu_{d}\right) \operatorname{tr} \varepsilon \mathbb{I}+2 \mu_{d} \varepsilon^{d}=\lambda \operatorname{tr} \varepsilon \mathbb{I}+2 \mu_{d} \varepsilon
$$

which is the usual constitutive equation of an isotropic elastic material, $\lambda$ and $\mu_{d}$ being its Lamé constants.

The constitutive equation (1) can be written:

$$
\sigma=\left(\lambda+\mu_{d}\right) \operatorname{tr} \varepsilon \mathbb{I}+\Sigma \frac{\varepsilon^{d}}{\left\|\varepsilon^{d}\right\|}
$$

with $\Sigma=2\left(\mu_{m}\left\|\varepsilon^{d}\right\|+\left(\mu_{d}-\mu_{m}\right) M\right)$. The coefficient $\mu_{m}$ being assumed to be negative, the plot of $\frac{\Sigma}{2}$ versus $\left\|\varepsilon^{d}\right\|$ is:

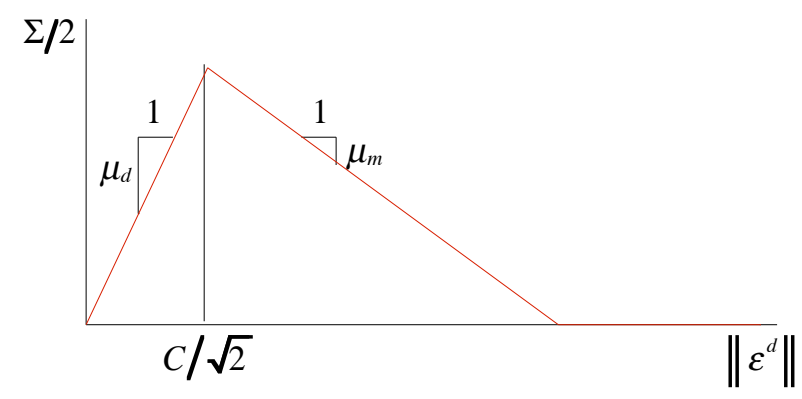


The relation $\left\|\varepsilon^{d}\right\| \rightarrow \Sigma$ which is the deviatoric strain stress relation for a fixed deviatoric strain direction $\frac{\varepsilon^{d}}{\left\|\varepsilon^{d}\right\|}$ is the same as that which is considered in [2] for 1D problems.

$\Sigma$ is clearly a continuous function of $\left\|\varepsilon^{d}\right\|$, so $\sigma$ is a continuous function of $\varepsilon$.

In the following $\Lambda$ denotes $\lambda+\mu_{d}$, so the constitutive equation (1) reads:

$$
\sigma=\Lambda \operatorname{tr} \varepsilon \mathbb{I}+2\left(\mu_{m}+\left(\mu_{d}-\mu_{m}\right) \frac{M}{\left\|\varepsilon^{d}\right\|}\right) \varepsilon^{d}
$$

and it is assumed that $0<\Lambda+\mu_{m}$.

\section{Axisymmetric problem and elementary solutions}

We consider the deformation at equilibrium of a circular annulus of inner aud outer radii $R_{i}$ and $R_{e}$ made of a material the constitutive law of which is (2). The inner and outer circles of the annulus are respectively subject to the pressures $p_{i}$ and $p_{e}$, no surface force density is applied to the annulus. As no orthoradial forces are applied on the boundaries, the orthoradial displacement $v$ is defined up to a constant, to fix this constant, $v$ is set to zero on the inner circle. The solution of the problem is looked for in the form:

$$
\mathbf{u}=u \mathbf{e}_{r}+v \mathbf{e}_{\theta} \text { with } \partial_{\theta} u=\partial_{\theta} v=0
$$

$r$ and $\theta$ are the polar coordinates of the space variable $\mathbf{x}$ and $\mathbf{e}_{r}$ and $\mathbf{e}_{\theta}$ are the associated basis vectors. From (3), we successively deduce that :

$$
\begin{aligned}
\varepsilon & =\partial_{r} u \mathbf{e}_{r} \otimes \mathbf{e}_{r}+\frac{1}{2}\left(\partial_{r} v-\frac{v}{r}\right)\left(\mathbf{e}_{\theta} \otimes \mathbf{e}_{r}+\mathbf{e}_{r} \otimes \mathbf{e}_{\theta}\right)+\frac{u}{r} \mathbf{e}_{\theta} \otimes \mathbf{e}_{\theta} \\
\operatorname{tr} \varepsilon & =\partial_{r} u+\frac{u}{r} \\
\varepsilon^{d} & =\frac{1}{2}\left(\left(\partial_{r} u-\frac{u}{r}\right)\left(\mathbf{e}_{r} \otimes \mathbf{e}_{r}-\mathbf{e}_{\theta} \otimes \mathbf{e}_{\theta}\right)+\left(\partial_{r} v-\frac{v}{r}\right)\left(\mathbf{e}_{r} \otimes \mathbf{e}_{\theta}+\mathbf{e}_{\theta} \otimes \mathbf{e}_{r}\right)\right) \\
\left\|\varepsilon^{d}\right\|^{2} & =\frac{1}{2}\left(\left(\partial_{r} u-\frac{u}{r}\right)^{2}+\left(\partial_{r} v-\frac{v}{r}\right)^{2}\right)
\end{aligned}
$$

The carrying of those relations in the constitutive equation (2) yields the stress field $\sigma$ in the form:

$$
\sigma=\sigma_{r r} \mathbf{e}_{r} \otimes \mathbf{e}_{r}+\sigma_{r \theta}\left(\mathbf{e}_{\theta} \otimes \mathbf{e}_{r}+\mathbf{e}_{r} \otimes \mathbf{e}_{\theta}\right)+\sigma_{\theta \theta} \mathbf{e}_{\theta} \otimes \mathbf{e}_{\theta}
$$

with:

$$
\sigma_{r r}=\Lambda\left(\partial_{r} u+\frac{u}{r}\right)+\left(\mu_{m}+\left(\mu_{d}-\mu_{m}\right) \frac{M}{\left\|\varepsilon^{d}\right\|}\right)\left(\partial_{r} u-\frac{u}{r}\right)
$$




$$
\begin{aligned}
\sigma_{\theta \theta} & =\Lambda\left(\partial_{r} u+\frac{u}{r}\right)-\left(\mu_{m}+\left(\mu_{d}-\mu_{m}\right) \frac{M}{\left\|\varepsilon^{d}\right\|}\right)\left(\partial_{r} u-\frac{u}{r}\right) \\
\sigma_{r \theta} & =\left(\mu_{m}+\left(\mu_{d}-\mu_{m}\right) \frac{M}{\left\|\varepsilon^{d}\right\|}\right)\left(\partial_{r} v-\frac{v}{r}\right)
\end{aligned}
$$

Due to the fact that the components of the stress do not depend on $\theta$, the equilibrium equation $\operatorname{div} \sigma=0$ reads:

$$
\left(\partial_{r} \sigma_{r r}+\frac{1}{r}\left(\sigma_{r r}-\sigma_{\theta \theta}\right)\right) \mathbf{e}_{r}+\left(\partial_{r} \sigma_{r \theta}+\frac{2}{r} \sigma_{r \theta}\right) \mathbf{e}_{\theta}=0
$$

The equation $\partial_{r} \sigma_{r \theta}+\frac{2}{r} \sigma_{r \theta}=0$ for $\sigma_{r \theta}$ integrates into $\sigma_{r \theta}=\frac{K}{r^{2}}$. That proves, together with the possible interface conditions on circles between softening and hardening parts of the material, that $\sigma_{r \theta}$ is continuous. Therefore, due to the boundary conditions $\left(\sigma_{r \theta}=0\right.$ for $r=R_{i}$ and $\left.r=R_{e}\right)$ :

$$
\sigma_{r \theta}=0
$$

That entails that either $\mu_{m}+\left(\mu_{d}-\mu_{m}\right) \frac{M}{\left\|\varepsilon^{d}\right\|}=0$, which can hold true only for $\frac{C}{\sqrt{2}} \leq\left\|\varepsilon^{d}\right\|$ and yields $\left\|\varepsilon^{d}\right\|=\frac{\mu_{d}-\mu_{m}}{-\mu_{m}} \frac{C}{\sqrt{2}}$, or $\partial_{r} v-\frac{v}{r}=0$ and $v=D r$.

Let's call elementary solutions the displacement fields that are solutions of the equation $\partial_{r} \sigma_{r r}+\frac{1}{r}\left(\sigma_{r r}-\sigma_{\theta \theta}\right)=0$ in some internal annulus of the studied domain. Three cases are to be considered, $\left\|\varepsilon^{d}\right\| \leq \frac{C}{\sqrt{2}}$ (and necessarily $v=D r$ ), $\frac{C}{\sqrt{2}} \leq\left\|\varepsilon^{d}\right\|$ with $v=D r$ and lastly $\left\|\varepsilon^{d}\right\|=\frac{\mu_{d}-\mu_{m}}{-\mu_{m}} \frac{C}{\sqrt{2}}$.

Type 1 elementary solution - case $\left\|\varepsilon^{d}\right\| \leq \frac{C}{\sqrt{2}}$.

This is the usual case of linear elasticity, we have $u=A_{1} r+\frac{B_{1}}{r}, v=D_{1} r$ and $\sigma_{r r}=2 \Lambda A_{1}-2 \mu_{d} \frac{B_{1}}{r^{2}}, A_{1}, B_{1}$ and $D_{1}$ being integration constants to be determined by interface or boundary conditions. In that case $\left\|\varepsilon^{d}\right\|=\frac{1}{\sqrt{2}}\left|\partial_{r} u-\frac{u}{r}\right|=\sqrt{2} \frac{\left|B_{1}\right|}{r^{2}}$ and this solution is valid in a annulus $R_{b} \leq r \leq R_{e}$ if $2 \frac{\left|B_{1}\right|}{R_{b}^{2}} \leq C$

Type 2 elementary solution - case $\frac{C}{\sqrt{2}} \leq\left\|\varepsilon^{d}\right\|$ and $v=D r$.

In that case we have $u=-\hat{C} s r \ln \frac{r}{A_{2}}+\frac{B_{2}}{r}, v=D_{2} r$ and $\sigma_{r r}=-\Lambda \hat{C} s \ln \frac{r^{2}}{A_{2}^{2}}-2 \mu_{m} \frac{B_{2}}{r^{2}} A_{2}$, $B_{2}$ and $D_{2}$ being integration constants and where $s=\operatorname{sgn}\left(\partial_{r} u-\frac{u}{r}\right)$ (sgn denotes the sign function) and $\hat{C}=\frac{\mu_{d}-\mu_{m}}{\Lambda+\mu_{m}} C$ (due to the hypothesis $0<\Lambda+\mu_{m}, \hat{C}$ is positive). 
We too have $\left\|\varepsilon^{d}\right\|=\frac{1}{\sqrt{2}}\left|\partial_{r} u-\frac{u}{r}\right|=\sqrt{2}\left(\frac{\left|B_{2}\right|}{r^{2}}-\hat{C}\right)$ and this solution is valid in a annulus $R_{b} \leq r \leq R_{e}$ if $C \frac{\Lambda+\mu_{d}}{\Lambda+\mu_{m}} \leq \frac{2\left|B_{2}\right|}{R_{e}^{2}}$.

Type 3 elementary solution - case $\left\|\varepsilon^{d}\right\|=\frac{\mu_{d}-\mu_{m}}{-\mu_{m}} \frac{C}{\sqrt{2}}$.

We have $u=A_{3} r+\frac{B_{3}}{r}, v= \pm \int \frac{1}{r^{3}}\left(\frac{\left(\mu_{d}-\mu_{m}\right)^{2}}{\mu_{m}^{2}} C^{2} r^{4}-4 B_{3}^{2}\right)^{1 / 2} \mathrm{~d} r+D_{3} r$ and $\sigma_{r r}=$ $2 \Lambda A_{3} . A_{3}, B_{3}$ and $D_{3}$ being integration constants. In that case $\left\|\varepsilon^{d}\right\|=\frac{\mu_{d}-\mu_{m}}{-\mu_{m}} \frac{C}{\sqrt{2}}$ and the validity condition of this solution comes down to the positivity of $\frac{\left(\mu_{d}-\mu_{m}\right)^{2}}{\mu_{m}^{2}} C^{2} r^{4}-$ $4 B_{3}^{2}$, in a annulus $R_{b} \leq r \leq R_{e}$, it reads $2 \frac{\left|B_{3}\right|}{R_{b}^{2}} \leq \frac{\mu_{d}-\mu_{m}}{-\mu_{m}} C$.

\section{Solutions of the boundary value problem}

The elementary solutions of section (3) satisfy the bulk equations (equilibrium and constitutive equation) but they still comprise integration constants. To form solutions of the boundary value problem, let's consider a succession of the elementary solutions defined in a successsion of concentric annuli and apply the boundary conditions as well as continuity conditions for the displacement and the radial stress $\sigma_{r r}$ at the interfaces between the concentric annuli.

Let's first consider the classical type 1 solution in the whole annulus $\left(R_{i} \leq r \leq\right.$ $\left.R_{e}\right)$. It is obviously purely radial $\left(D_{1}=0\right)$ and the other integration constants $A_{1}$ and $B_{1}$ are determined using the boundary conditions. According to section (3) this solution is valid for $\left|p_{i}-p_{e}\right| \leq C \mu_{d} \frac{R_{e}^{2}-R_{i}^{2}}{R_{e}^{2}}$.

For $C \mu_{d} \frac{R_{e}^{2}-R_{i}^{2}}{R_{e}^{2}}<\left|p_{i}-p_{e}\right|$, the previous classical solution is no more valid since in points near the inner circle, the norm $\left\|\varepsilon^{d}\right\|$ of the deviatoric part of the strain excceds the threshold $\frac{C}{\sqrt{2}}$. We then consider a solution made of two elementary solutions, one of type 2 , the other of type 1 in two concentric annuli. The 6 corresponding integration constants are determined using the boundary and the interface continuity conditions. It turns out that that solution is purely radial $(v=0)$ and that the validity conditions for the two elementary solutions entail that, in the inner annulus $\left(R_{i} \leq r \leq R_{1}\right)$, the solution is of type 2 and of type 1 in the outer annulus $\left(R_{1} \leq r \leq R_{e}\right)$, the radius of the interface between the 2 annuli being solution of the transcendental equation:

$$
\begin{aligned}
\mid\left(\Lambda+\mu_{m}\right) \mu_{d} R_{i}^{2} & \left(R_{e}^{2}-R_{1}^{2}\right)+\left(\Lambda+\mu_{d}\right) \mu_{m} R_{e}^{2}\left(R_{1}^{2}-R_{i}^{2}\right) \mid C \\
& =\left(\Lambda+\mu_{m}\right) R_{i}^{2} R_{e}^{2}\left|p_{e}-p_{i}-2 \Lambda \hat{C} s \ln \frac{R_{1}}{R_{i}}\right|
\end{aligned}
$$


the solving of which can only be performed numerically.

The previous solution is the only purely radial solution in the case $C \mu_{d} \frac{R_{e}^{2}-R_{i}^{2}}{R_{e}^{2}}<$ $\left|p_{i}-p_{e}\right|$. That does not mean that that solution is unique, indeed bifurcation occurs in the form of non radial solutions, therefore showing shear bands. To prove that, we consider a solution made of a succession of a type 2, a type 3 and a type 2 elementary solutions in three concentric annuli. Due to the same validity conditions as for the purely radial solution, the type 2 solution has to take place in a more inner annulus than that of the type 1 solution. Here we consider a solution which is of type 2 in the inner annulus $\left(R_{i} \leq r \leq R_{1}\right)$, of type 3 in the intermediate annulus $\left(R_{1} \leq r \leq R_{2}\right)$ and of type 2 in the outer annulus $\left(R_{2} \leq r \leq R_{e}\right)$.

Due to the condition $v\left(R_{i}\right)=0$ on the orthoradial displacement, the type 2 solution in the inner annulus is purely radial but the orthoradial displacement $v$ is necessarily different from 0 in the type 3 intermediate annulus and, by continuity condition, so it is in the type 1 outer annulus. The intermediate annulus can be seen as a shear band.

Contrary to the case of the purely radial solution, the validity conditions of the three elementary solutions do not sum up in an equality but in a set of inequalities for the integration constant $B_{2}$ of the type 2 solution:

$$
C R_{1}^{2} \leq 2\left|B_{2}\right| \leq \min \left(\frac{\Lambda}{\Lambda+\mu_{d}} \frac{\mu_{d}-\mu_{m}}{-\mu_{m}} C R_{1}^{2}, C R_{2}^{2}\right)
$$

That shows that, most probably, the values of the two radii $R_{1}$ and $R_{2}$ are not completely determined but are bounded by some inequalities. This is consistent with the expected fact that the width of the shear band (the intermediate annulus $\left(R_{1} \leq r \leq R_{2}\right)$ is not determined as there is no internal length in the modeling.

\section{Conclusion}

Considering the deformation of a hardening/softening "elastic" material in a 2D annulus submitted to inner and outer pressures, which enables the determination of anlytical solutions, it has been proved that, past the softening threshold, the purely radial solution remains unique but that shear banding may occur. The analytical solutions with shear banding have been determined bringing possible benchmark for numerical simulation. It has also been confirmed that the shear bands have no determined length, as expected owing to the lack of any intrisic length in the modeling.

\section{References}

1. Budiansky B., A Reassment of Deformation Theories of Plasticity. J. of Applied Mechanics, 1959, pp. 259-264. 
2. Chambon R, Caillerie D, El Hassan N., One dimensional localization studied with a second grade model. European Journal of Mechanics A/Solids, vol. 17 No 4 1998, pp. 637-656.

3. Chambon R, Crochepeyre S, Charlier R., An algorithm and a method to search bifurcation points in non-linear problems. International Journal for Numerical Methods in Engineering vol. 51 2001, pp. 315-332.

4. Desrues J., Tracking Strain Localization in Geomaterials Using Computerized Tomography. Proc. of the International Workshop on X-ray CT for Geomaterials Kumamoto, Japan, November 6-7 2003. Balkema, Lisse, The Netherlands, 2004, pp: 15-41.

5. Desrues J., Viggiani G., Strain localization in sand : an overview of the experimental results obtained in Grenoble using stereophotogrammetry, Int. Journal for Numerical and Analytical methods in Geomechanics vol. 28 No 4 2004, pp. 279-321.

6. Rice J., The localization of plastic deformation. In International Congress of Theoretical and Applied Mechanics. Koiter WD (ed.), North Holland, Amsterdam, 1976.

7. Rudnicki J.W., Rice J.R., Conditions for the localization of deformation in pressure-sensitive dilatant materials, J. Mech. Phys. Solids vol. 23 1975, pp. 371-394. 